\title{
Errata
}

\section{Erratum: Graupner et al., "Natural Firing Patterns Imply Low Sensitivity of Synaptic Plasticity to Spike Timing Compared with Firing Rate"}

In the article "Natural Firing Patterns Imply Low Sensitivity of Synaptic Plasticity to Spike Timing Compared with Firing Rate" by Michael Graupner, Pascal Wallisch, and Srdjan Ostojic, which appeared on pages 11238-11258 of the November 2, 2016 issue, there is a production error in Table 1 and its caption. " $\mathrm{A}_{3}{ }^{-}$" should read " $\mathrm{A}_{3}{ }^{+}$” in the Parameter column and caption. This error does not affect the conclusions of the paper. The article has been corrected online and the table appears below.

\begin{tabular}{|c|c|c|}
\hline Parameter & Unit & Value \\
\hline $\mathrm{A}_{2}^{+}$ & & $0^{b}$ \\
\hline$\tau_{+}$ & $\mathrm{ms}$ & $16.8^{b}$ \\
\hline $\mathrm{A}_{2}^{-}$ & & 0.00826477 \\
\hline$\tau_{-}$ & $\mathrm{ms}$ & $33.7^{b}$ \\
\hline $\mathrm{A}_{3}^{+}$ & & 0.0165746 \\
\hline$\tau_{\mathrm{y}}$ & $\mathrm{ms}$ & 56.38234 \\
\hline
\end{tabular}

DOI: 10.1523/JNEUROSCI.0051-19.2019

\section{Erratum: Takata et al., "Sleep and Wakefulness Are Controlled by Ventral Medial Midbrain/Pons GABAergic Neurons in Mice"}

In the article "Sleep and Wakefulness Are Controlled by Ventral Medial Midbrain/Pons GABAergic Neurons in Mice" by Yohko Takata, Yo Oishi, Xu-Zhao Zhou, Emi Hasegawa, Koji Takahashi, Yoan Cherasse, Takeshi Sakurai, and Michael Lazarus, which appeared on pages 10080-10092 of the November 21, 2018 issue, Figure 2Q appeared incorrectly. This correction does not affect the conclusions of the paper. The article has been corrected in the online and a corrected version of the figure is displayed below. 

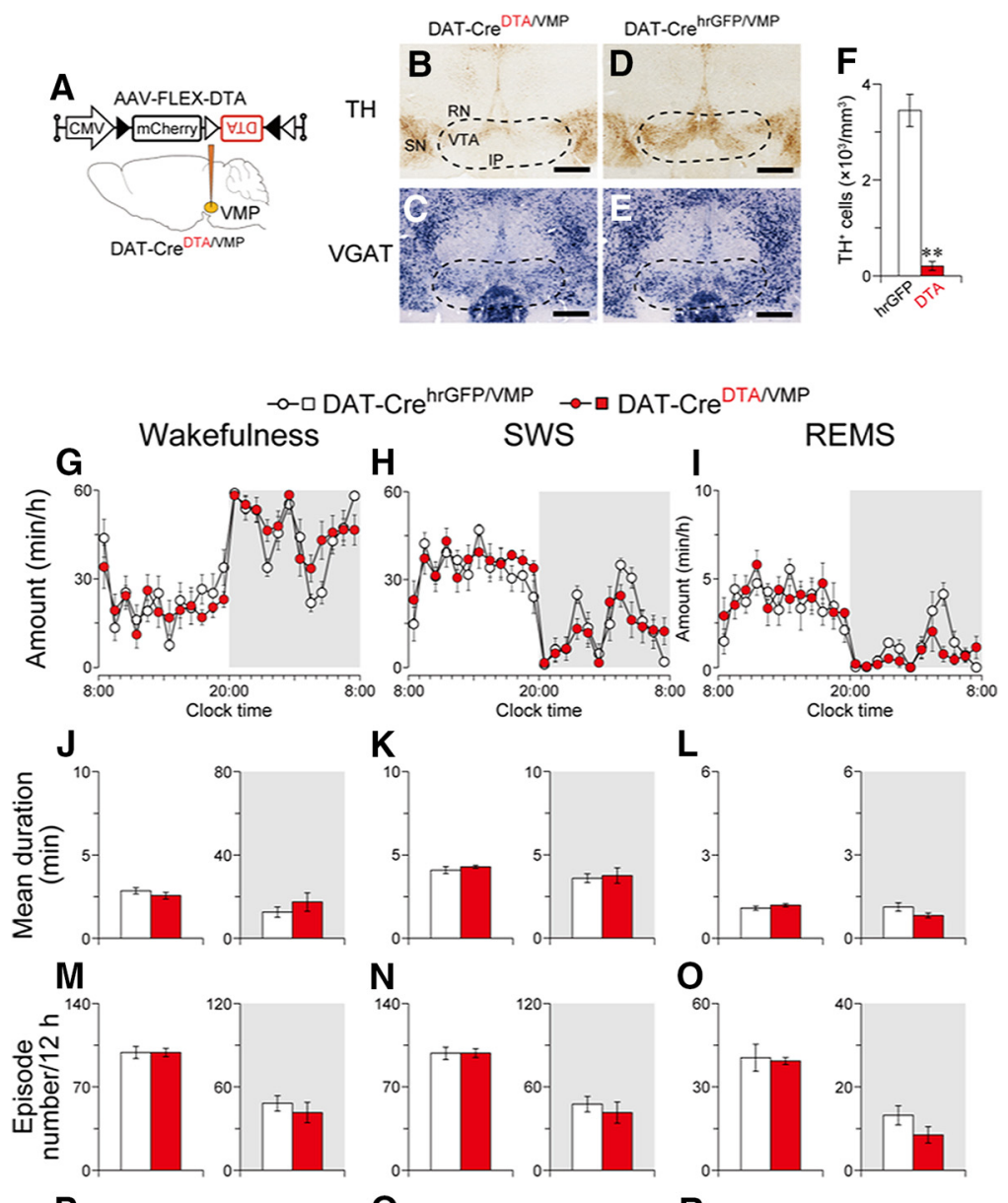

K
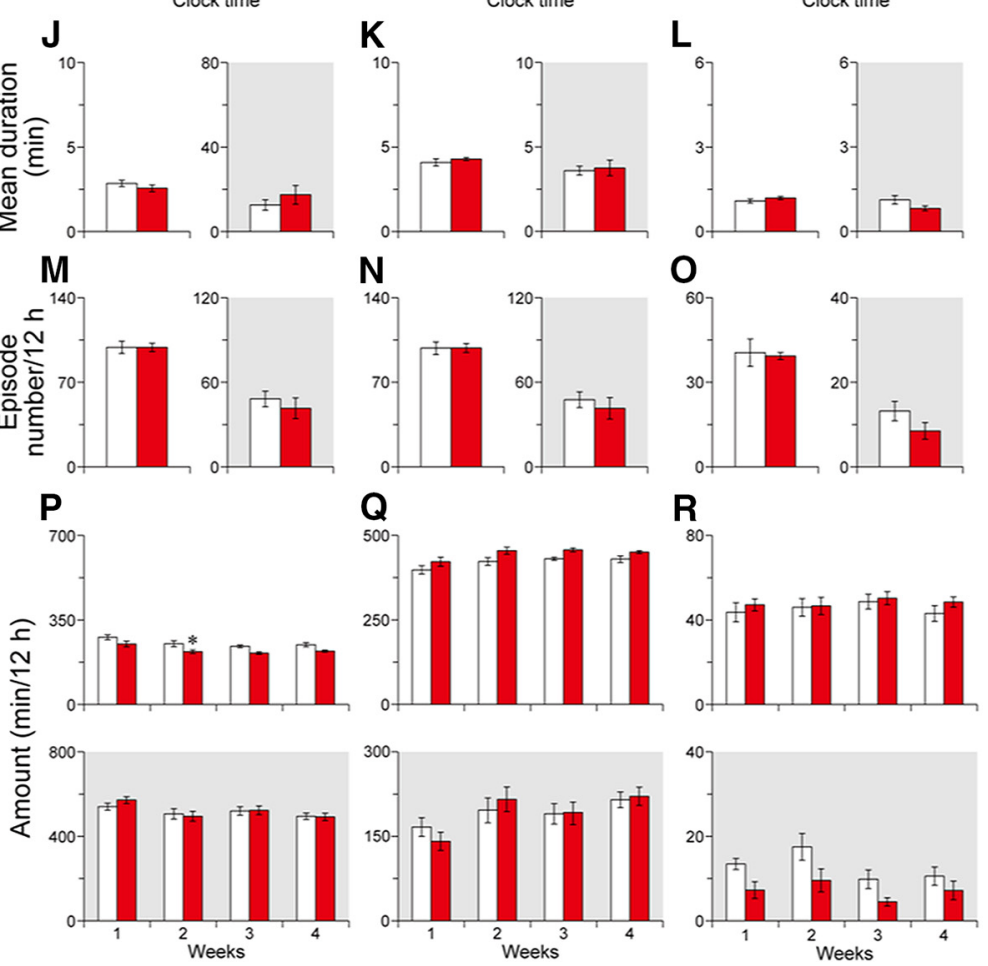

Figure 2.

DOI: 10.1523/JNEUROSCI.0053-19.2019 